\title{
Plasmid to generate Mycobacteria mutants
}

\author{
Qi Deng, Jianzhou Meng*, Yan Guan, Yishuang Liu and Chunling Xiao
}

\begin{abstract}
The generation of conditional mutants has been an effective approach to studying bacteria and validating drug targets, and mutants of Mycobacteria are no exception. However unlike other bacteria, there is still a paucity of available tools for Mycobacteria. We constructed a new plasmid containing tetracycline-repressive expression system (TetRr1.7) and Xer Site-Specific recombinase system to generate label-free controllable expression strains. The plasmid was subsequently used to construct a strain of $M$. tuberculosis expressing the only copy of D-alanine:D-alanine ligase under the control of the tetracycline-repressive promoter. The results showed that the mutant strain lost the ability of colony formation, became more sensitive to D-cycloserine and the cell wall of the mutant strain was disrupted when anhydrotetracycline was added to the medium. Taken together these observations, confirmed that the expression of D-alanine:D-alanine ligase was tightly controlled by the promoter. In conclusion, the new plasmid is a convenient tool for constructing stable conditional mutant strains in Mycobacteria and can be used for future target identification.
\end{abstract}

Keywords: Conditional mutant, Tetracycline-inducible expression system, D-alanine:D-alanine ligase, Mycobacterium tuberculosis, Plasmid construction

\section{Introduction}

Tuberculosis (TB) caused by Mycobacterium tuberculosis $(M t b)$ still remains a heavy threat to human beings. There were about 10 million patients who developed into tuberculosis and 1 million death every year, many of which were MDR-TB (Kwon 2017). There is an urgent need for new drugs and vaccines to treat the disease. However, the efforts to exploit new anti-TB drugs seemed hopeless as during the last 50 years only two agents were approved (Lu et al. 2014; Blair and Scott 2015). Thus far only a limited number of targets could be used to develop the drugs, which explains the low success rate. Further the identification of new targets was difficult because of the lack of tools to research the molecular microbiology of $M t b$.

The plasmids used for molecule manipulation in $\mathrm{Myco-}$ bacteria limited to pMind, pMV261, pMY769, pAZI9479, pNIL-pGOAL and so on. Plasmids pMind, pMV261 and pMY769 can carry the DNA fragment into cell, but the

\footnotetext{
*Correspondence: mengjianzhou@126.com Institute of Medicinal Biotechnology, Chinese Academy of Medical Sciences and Peking Union Medical College, Beijing, China
}

gene dosage cannot be quantified as these plasmids cannot integrate into the genome or another copy of the gene exists in the host genome and cannot be controlled by the operon of the plasmid. Plasmid pAZI9479, a suicide plasmid for mycobacteria, can replace the promoter of the target gene with the ptr promoter through homologous recombination. However the mutant strain is not stable and can reverse to the wild type. Plasmid pNIL-pGOAL can knockout a target gene through double homologous recombination. However, in our hands, knockout strains of the essential genes could not be obtained and the target genes were cutoff so we could only study at the deficiency conditions. Inducible systems used in mycobacteria are also problematic. Currently, the mainly reported systems contain: acetamidase inducible system, pristinamycin inducible system, IPTG inducible system, nitrilases inducible system, theophylline riboswitch system and tetracycline inducible system. The acetamideinducible system is the earliest mycobacterial inducible system described, which is used for over-expression, but the basal activity of promoter is high and prone to recombination (Parish et al. 1997; Brown and Parish 2006). Pristinamycin inducible system, IPTG inducible system 
are also successfully developed for gene regulation in mycobacteria (Forti et al. 2009; Ravishankar et al. 2015). IPTG inducible system is more frequently used in $E$. coli expression and follow-up applications in mycobacterium are limited. There are also references about nitrilases inducible system and theophylline riboswitch system (Pandey 2009; Seeliger et al. 2012). Tetracycline inducible system is the most widely used and has been validated that they can be used to regulate gene expression in animal models of infection (Carroll et al. 2005; Ehrt et al. 2005a, b; Hernandez-Abanto et al. 2006). Considering all the above, we endeavoured to design a new plasmid containing tetracycline inducible system with the following advantageous characteristics that allow the researcher to: (i) construct a stable mutant strain; (ii) control gene expression quantitatively through the promoter of the plasmid.

Herein we describe the successful construction of a new plasmid containing a TetRr1.7, a tetracycline-repressive expression system which has been shown to express a target gene expression quantitatively in the presence of the repressor (tetracycline or anhydrotetracycline) (Guo et al. 2007). Target gene expression was under the control of a promoter which could bind with the TetRr1.7anhydrotetracycline complex. Herein we also validate the plasmid and illustrate that we could indeed regulate the gene expression of D-alanine:D-alanine ligase (Ddl) in Mycobacteria.

\section{Materials and methods}

\section{Bacterial strains and media}

Mycobacterium tuberculosis $\mathrm{H}_{37} \mathrm{Rv}$ (ATCC27294) was cultured on Middlebrook 7H10 agar media supplemented with Oleic Albumin Dextrose Catalase (OADC) (Allen 1998; Hodgkinson et al. 2015) or in 7H9 broth plus OADC and polysorbate 80. Escherichia coli DH5 $\alpha$ (TransGen Biotech, Beijing, China) was cultured in Luria-Bertani (LB) broth or on LB agar medium. Kanamycin (Amresco, Bedfordshire, UK) was added at the concentration of $100 \mu \mathrm{g} \mathrm{mL}^{-1}$ for $E$. coli and hygromycin
(Amresco, Bedfordshire, UK) $100 \mu \mathrm{g} \mathrm{mL} \mathrm{m}^{-1}$ for Mycobacteria. Anhydrotetracycline (Sigma-Aldrich, Germany) was added as appropriate. Plasmids used are listed in Table 1.

\section{Plasmid constructions}

Genomic DNA was extracted from $M t b \mathrm{H}_{37} \mathrm{Rv}$ in the logarithmic phase and used as PCR template, as previously described (van Helden et al. 2001). PCR conditions were as follows: hot start at $94{ }^{\circ} \mathrm{C}$ for $10 \mathrm{~min}$, followed by 30 cycles of $94{ }^{\circ} \mathrm{C}$ for $40 \mathrm{~s}, 60{ }^{\circ} \mathrm{C}$ for $30 \mathrm{~s}$, and $72{ }^{\circ} \mathrm{C}$ for 30-120 s (depending on the fragment length), and a final extension at $72{ }^{\circ} \mathrm{C}$ for $10 \mathrm{~min}$. Plasmid pMind was digested with Nhe-I to remove pAL5000 fragment, the replicon of Mycobacteria, and to obtain pIMBS. Promoter $P_{m y c 1}$ tetO (Ehrt et al. 2005a, b) was chosen to control the target gene expression in Mycobacteria and was synthesized chemically by Ruibiotech (Beijing, China). $P_{\text {myc } 1}$ tet $O$ and pIMBS were digested with Hind-III and Spe-I. After ligation pIMBSt was obtained and which also no longer contained the hygromycin resistant gene. The streptomycin resistance gene was amplified using primers SmdifS and SmdifR from pJRD215, and cloned between the $\mathrm{Xba}-\mathrm{I}$ and $\mathrm{BamH}$-I sites of pIMBSt to get pIMBSs in which two dif sites of $M t b$ lineated in the primers that were added to excise the resistance gene in Mycobacteria. Then the hygromycin resistance gene was amplified from pMind using HygroS and HygroR primers to substitute the streptomycin resistance, obtaining pIMBSh in which the hygromycin resistance gene was controlled by the promoter Ptet 2 . A fragment about $370 \mathrm{bp}$ of pMY769 was amplified using primers Gap1S and Gap1R, and the product was cloned into the BamH-I and Spe-I sites of pIMBSh to separate the $\mathrm{P}_{\text {mycl }}$ tetO and the downstream operon, obtaining pIMBSg. Primers P85S and P85R were used to amplify promoter $p A G 85$ from pGOAL19, while PeS and PeR amplified eGFP from pEGFP-C1. Then, both PCR products were mixed as templates and primers P85S and PeR were used to synthesize a whole fragment containing promoter and eGFP. The fragment was

Table 1 Plasmids used in this study

\begin{tabular}{lll}
\hline Plasmids & Application & Source \\
\hline pMind & Used to construct pMDX and as template to amplify hygromycin resistance gene & Robertson, Brian D (Blokpoel et al. 2005) \\
pJRD215 & Used as template to amplify streptomycin resistance gene & Professor Xiangmei Liu (Chen et al. 2011) \\
pMY769 & Used as template to amplify gap fragments & Professor Francesca Forti (Forti et al. 2009) \\
pGOAL19 & Used as template to amplify the promotor pAG85 & Tanya Parish (Parish and Stoker 2000) \\
pEGFP-C1 & Used as template to amplify EGFP gene & Doc Zhang (Zhang et al. 1996) \\
pJV53 & Used to facilitate recombination & van Kessel, J.C. (van Kessel and Hatfull 2007) \\
pMDX & The plasmid tool & This study \\
\hline
\end{tabular}


cloned using $P f l \mathrm{~m}$-I and Spe-I sites of pIMBSg to obtain pIMBSe. Primers gap2S and gap2R were used to amplify fragment from pMY769 and the product was cloned into Spe-I digested pIMBSe through seamless cloning, getting pIMBSeg. Part of the TetRr 1.7 was synthesized and cloned using the Xba-I and Kpn-I sites of pIMBSeg to generate pIMBSr. Primers PfurA102S and PfurA102R were used to amplify promoter PfurA102 from pMY769, and cloned into pIMBSr digested by $\mathrm{Xba}$-I through seamless cloning, thereby obtaining pMDX. All primer sequences used above are listed in Additional file 1.

\section{Construction of the promoter replacement mutants in Mtb}

To construct $M t b D d l$ mutant strain, the forward fragment and fragment after initiation codon ATG (about $400 \mathrm{bp}$ ) of the target genes were separately amplified by primers $\mathrm{d} 1 \mathrm{~S}, \mathrm{~d} 1 \mathrm{R}$ and $\mathrm{d} 2 \mathrm{~S}, \mathrm{~d} 2 \mathrm{R}$ (listed in Additional file 1 ) and then cloned into PMDX to construct pMDXD. The competent $M t b$ cells and plasmids were prepared as described (Hinds et al. 1999; Parish et al. 1999). In brief, the cells were collected and washed three times with $10 \%$ glycerol reducing the volume each time. They were then suspended in $1 / 500$ of the initial volume using ice-cold $10 \%$ glycerol. In order to transform the competent cells, $5 \mu \mathrm{L}$ pJV53 (no more than $1 \mu \mathrm{g}$ ) was added to $200 \mu \mathrm{L}$ competent cells, and transferred to a $0.2 \mathrm{~cm}$ cuvette (Biorad, Hercules, CA, USA). The electroporation program used was: voltage $2.5 \mathrm{kV}$, capacitance $25 \mu \mathrm{F}$, resistance $1000 \Omega$, and a pulse time of $15-25 \mathrm{~ms}$. The pulsed cells were cultured in $5 \mathrm{~mL} 7 \mathrm{H} 9$ broth for $24-48 \mathrm{~h}$ at $37^{\circ} \mathrm{C}$, spread on $7 \mathrm{H} 10$ agar containing $100 \mu \mathrm{g} \mathrm{mL}^{-1}$ kanamycin and incubated at $37{ }^{\circ} \mathrm{C}$ until bacterial colonies formed. The positive strain was confirmed by colony PCR using primer $\mathrm{kmS}$ (see Additional file 1) and primer $\mathrm{kmR}$ (see Additional file 1). The Mtb:pJV53 were cultured in 7H9 broth, containing $20 \mu \mathrm{g} \mathrm{mL}{ }^{-1}$ kanamycin and $0.2 \%$ (w/v) succinate at $37{ }^{\circ} \mathrm{C}$, and $0.2 \%(\mathrm{w} / \mathrm{v})$ acetamide was added to the culture for another $24 \mathrm{~h}$ (van Kessel and Hatfull 2007). The competent cells were prepared as before and electro-transformed with Hind-III and Pci-I digested pMDXD. The pulsed cells were refreshed and then spread on the $7 \mathrm{H} 10$ agar containing $100 \mu \mathrm{g} \mathrm{mL} \mathrm{L}^{-1}$ hygromycin and incubated at $37{ }^{\circ} \mathrm{C}$ until bacterial colonies formed. The bacteria were subsequently transferred to $7 \mathrm{H} 9$ liquid medium without antibiotic and incubated at $37{ }^{\circ} \mathrm{C}$ to allow them to excise the hygromycin resistance gene. The genome of the mutant was sequenced to ensure the successful insertion of the DNA fragments into the $M t b$ genome. The positive mutant strain was named Mtb::Ddl.

\section{Mutant colony formation}

The mutant strain and wild-type strain were cultured separately in $7 \mathrm{H} 9$ broth at different concentrations $(0$,
2, 20, 200, $2000 \mathrm{ng} \mathrm{mL}^{-1}$ ) of anhydrotetracycline. Until $\mathrm{OD}_{600}$ reached 0.55 , the bacterial suspensions were diluted 100 times and then spotted on 7H10 agar media in six-well plates. The plates were incubated at $37{ }^{\circ} \mathrm{C}$ to observe colony formation.

\section{Alterations in cell-wall contents of the mutant}

The mutant strain was cultured in $7 \mathrm{H} 9$ medium in the presence of different concentrations of anhydrotetracycline $\left(0,2,20,200,2000 \mathrm{ng} \mathrm{mL} \mathrm{m}^{-1}\right)$ for 2 weeks. The wild type strain was cultured in $7 \mathrm{H} 9$ medium with $\mathrm{D}$-cycloserine added at the concentration of $12 \mu \mathrm{g} \mathrm{mL}^{-1}$ for 2 weeks. Cell wall of Mycobacteria was extracted as described previously (Besra 1998; Meng et al. 2015).

\section{MIC of D-cycloserine to the mutant}

The minimum inhibitory concentration (MIC) was determined using the broth dilution method. Briefly, the mutant strain and wild-type strain were cultured in $7 \mathrm{H} 9$ medium in 96-well microtiter plates containing different concentrations of anhydrotetracycline $\left(0,2,20 \mathrm{ng} \mathrm{mL}^{-1}\right)$. A two-fold serial dilution of D-cycloserine was added, giving final concentrations of $128,64,32,16,8,4,2,1$, 0.5 and $0.25 \mu \mathrm{g} \mathrm{mL}^{-1}$. Rifampicin and isoniazid were used as control and the final concentrations of these two drugs were $64,32,16,8,4,2,1,0.5,0.25,0.125,0.0625$ and $0.03125 \mu \mathrm{g} \mathrm{mL}^{-1}$.

\section{The genetic stability of the mutant}

The mutant was cultured in $7 \mathrm{H} 9$ broth and inoculated once every 10 days. After fifty generations, the genome was extracted and the segment we inserted was sequenced.

\section{Results}

The plasmid pMDX with illustrated operon and restriction sites is shown in Fig. 1. To control the expression of the target gene in $M t b$, its upstream sequence should be cloned at the Kpn-I site anticlockwise, and the downstream sequence anticlockwise at Bst-XI site, with the ATG initiation codon of the gene at Bst-XI site. The lineated plasmid can conduct homologous recombination twice to substitute the promoter of the target gene with $P_{m y c t} t e t O$, and the expression of $P_{\text {myc }}$ tet $O$ is controlled by the repressor TetR-r1.7. The expression of target gene can therefore be repressed by anhydrotetracycline.

\section{Colony formation efficiency of the mutant}

Given that the $D d l$ gene is essential for Mycobacterium, the ability to form bacterial colonies was investigated in order to verify that the expression of $D d l$ was controllable. Different concentrations of anhydrotetracycline were therefore added to the culture of Mtb::Ddl strain 




Fig. 1 The map of the pMDX

harbouring the pMDXD plasmid, and colony formation on 7H10 agar media was recorded. Mutant and wild-type strains were equally efficient at forming colonies in the presence of $0-2 \mathrm{ng} \mathrm{mL}^{-1}$ anhydrotetracycline (Fig. 2). At $20 \mathrm{ng} \mathrm{mL}^{-1}$ anhydrotetracycline, the mutant strain could form colonies but there were fewer colonies than for the wild type strain (Fig. 2). And, strains cultivated in media containing 200 and $2000 \mathrm{ng} \mathrm{mL} \mathrm{L}^{-1}$ anhydrotetracycline formed more less bacterial colonies (Fig. 2). The results show that anhydrotetracycline did not affect the colony formation of wild type Mycobacterium. Also the mutant strain can form colony without anhydrotetracycline, but colony formation is impeded with increasing concentrations of anhydrotetracycline. These data indicate that expression of $D d l$ decreases when anhydrotetracycline is added.

\section{Alterations in the cell wall of the conditional mutant} $D d l$ catalyzes the ATP-driven ligation of two D-alanine (D-Ala) molecules to form the D-alanyl:D-alanine dipeptide, a key building block in peptidoglycan (Bruning et al. 2011). Peptidoglycan is critical to the integrity of the bacterial cell wall. It was therefore inferred that suppressing the expression of $D d l$ will result in the disruption of the bacterial cell wall. D-cycloserine is a second-line antiTB drug, the mechanism of which is to block peptidoglycan synthesis. In theory, the effect of anhydrotetracycline to the mutant strain is similar to the effect of D-cycloserine to the wild type strain. Thus, we also treated $M t b$ $\mathrm{H}_{37} \mathrm{Rv}$ with a sublethal concentration of D-cycloserine. The whole cell wall was extracted from $M t b \mathrm{H}_{37} \mathrm{Rv}$ and $M t b:: D d l$ in the plateau growth phase cultured in $7 \mathrm{H} 9$ containing anhydrotetracycline or D-cycloserine. The ratio of cell wall mass to whole cell mass of both strains is shown in Fig. 3. Without anhydrotetracycline, the mutant cell wall content was similar to that of the wild-type. The ratio of cell wall mass to whole cell mass reduced with increasing concentrations of anhydrotetracycline. For example, a ratio of 0.13 when $200 \mathrm{ng} \mathrm{mL} \mathrm{m}^{-1}$ anhydrotetracycline was added to the culture suggests that the mutant cells lost an average of $73.7 \%$ cell wall materials. When $12 \mu \mathrm{g} \mathrm{mL}{ }^{-1} \mathrm{D}$ - cycloserine was added, the ratio of cell wall mass to whole cell mass was 0.35 , which indicated that an average of $35 \%$ cell wall was lost. These data 


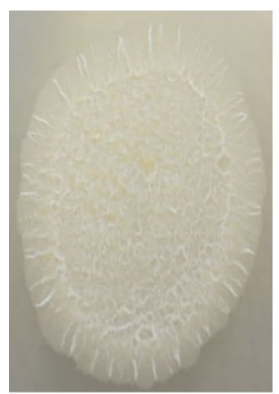

ATC 0ng mL-1

2000ng mL-1

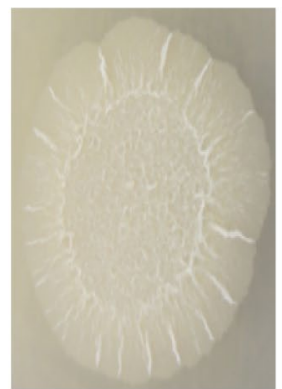

2ng mL-1

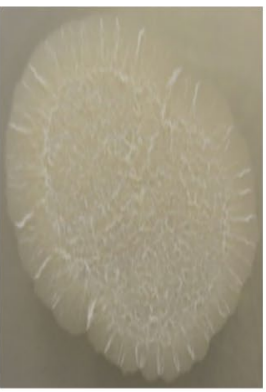

20ng mL-1

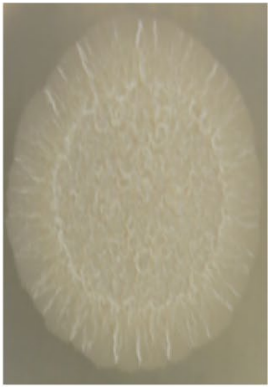

200ng mL-1

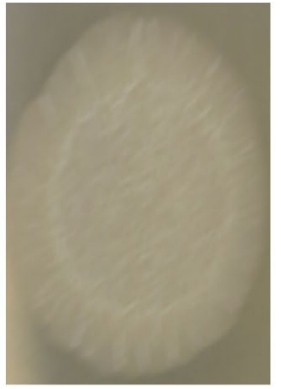

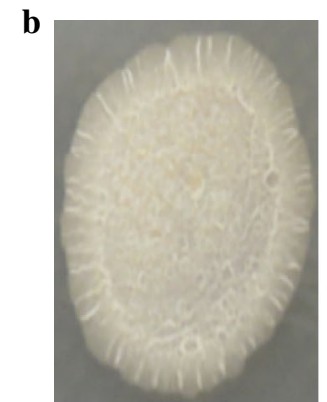
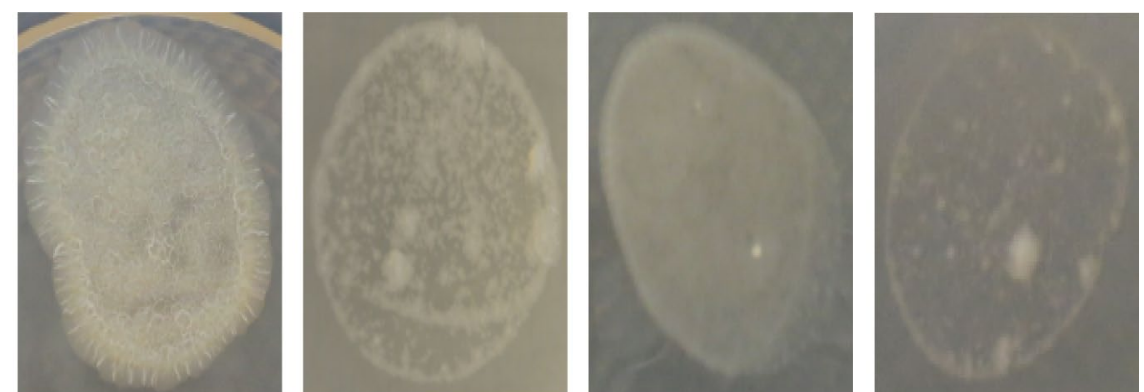

Fig. 2 Bacterial colony formation of the wild-type strain (a) and mutant strain (b) at different concentration of anhydrotetracycline

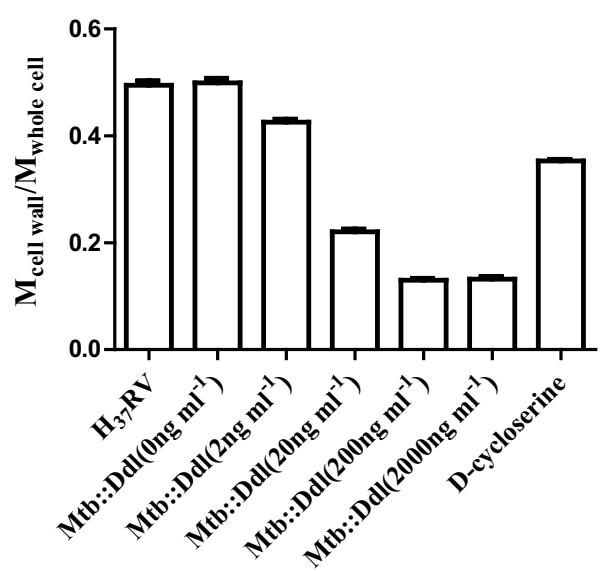

The strain type

Fig. 3 The proportion of the cell wall mass to the whole cell mass. The whole cell wall was extracted from wild-type H37Rv and Mtb::Ddl cultured in the presence of $0,2,20,200,2000 \mathrm{ng} \mathrm{mL}^{-1}$ anhydrotetracycline

confirmed that the expression of $D d l$ can be regulated using anhydrotetracycline, affecting Ddl protein level. Treated the wild type strain with D-cycloserine could have the same effect with treated the mutant strain with anhydrotetracycline.

\section{MIC of D-cycloserine to the mutant}

The MIC of D-cycloserine to the mutant Mtb::Ddl was determined, as D-cycloserine is a known inhibitor of Ddl. The MIC of D-cycloserine to the mutant was twice the MIC to wild-type strain. This may because the promoter $P_{m y c l}$ tet $O$ was stronger than its original promoter. The MIC of D-cycloserine to the mutant almost remained unchanged when the concentration of anhydrotetracycline was $2 \mathrm{ng} \mathrm{mL}^{-1}$. When $20 \mathrm{ng} \mathrm{mL}-1$ anhydrotetracycline was added to the media, the MIC of $\mathrm{D}$-cycloserine to the mutant was $1 \mu \mathrm{g} \mathrm{mL} L^{-1}$, while the MIC to wild type strain was $16 \mu \mathrm{g} \mathrm{mL}^{-1}$. The MICs of rifampicin and isoniazid to the mutant were both $0.0625 \mu \mathrm{g} \mathrm{mL}^{-1}$ when 0,2 and $20 \mathrm{ng} \mathrm{mL}^{-1}$ anhydrotetracycline was added, which were the same to the MICs of rifampicin and isoniazid to wild type strain. These data confirmed again that the anhydrotetracycline can repress the expression of $D d l$.

\section{The genetic stability of the mutant}

Genetic stability is one of the shortcomings of the existing plasmids for gene manipulation in Mycobacteria. To test whether the mutant can be genetic stable, the mutant was subcultured and the segments we inserted of the fiftieth generation was sequenced. The result confirmed that the mutant gene remained unchanged. 


\section{Discussion}

Developing tools for conditional gene regulation is of great importance to gene function research and drug target identification in bacteria. Experimental control of target gene expression is desired and can be achieved by replacing the native promoter of the target gene with a tightly regulated promoter whose activity can be controlled by the researcher (Gomez and Bishai 2000; Chalut et al. 2006; Gandotra et al. 2007). To date, the plasmids for promoter replacement in Mycobacteria are limited and have disadvantages. In this study a new plasmid, pMDX, was constructed which can be used to generate a stable mutant strain and control gene expression quantitatively as demonstrated using D-alanine:D-alanine ligase(Ddl) as a subject for target gene regulation in Mycobacteria.

The plasmid pMDX contains a regulated mycobacterial expression system TetRr-1.7 that permits the silencing of a gene by the addition of anhydrotetracycline to the culture medium. Regulation of gene repression by TetR-r1.7 has been validated through silencing secA1 in Mycobacterium smegmatis (Guo et al. 2007) and prcBA in M. tuberculosis (Gandotra et al. 2007). As high-level TetR expression is required when repression occurs, the promoter PfurA102 was used to control the expression of TetR-r1.7. The target gene was operated by Pmyc1tetO containing two tetO2 which can bind to TetRr1.7-anhydrotetracycline complex to shut down gene expression. For mutant strain screening, the hygromycin resistance gene was introduced into the plasmid, but it was nulled after obtained the mutant strains. This screening marker would otherwise lead to erroneous judgements especially during inhibitor screening and can affect the growth of the bacteria. Hence two dif fragments were added to both sides of the resistance gene, which were recognized and cut-out by Xer recombinases, obtaining the label-free mutant strains (Cascioferro et al. 2010). The eGFP gene was also cloned into the plasmid to monitor the growth of the bacteria in macrophage by detecting its fluorescence. This function still needs to be validated in a further study.

Another obstacle for conditional mutant strain construction in Mycobacteria is the low rate of homologous recombination. Gordhan and Parish described three methods to pretreat DNA to enhance the homologous recombination (Gordhan and Parish 2001). Singlestranded phagemid DNA can abolish unwanted recombination but the problem is the generation of sufficient amount of single-stranded DNA especially for the GC rich $M t b$ genome. UV pretreated DNA can raise the homologous recombination events, however, additional point mutations are inevitable. Alkali-denaturation will lead to no additional mutations but lower the homologous recombination events. Lineated double-stranded
DNA may also cause higher homologous recombination probability, but lineated DNA is not stable in bacteria. Plasmid pJV53, an Escherichia-Mycobacteria shuttle plasmid, can generate Che9c mycobacteria phage gp60 and gp61 proteins, which are homologous to recombinant protein RecE and RecT respectively. These recombinant proteins promote foreign genes to recombine with homologous genes in Mycobacteria with high efficiency (van Kessel and Hatfull 2007). In this study, before the lineated DNA was introduced into $M t b$, plasmid pVJ53 was therefore electroporated into $M t b$ to improve homologous recombination. Only the double cross-over mutants could form colonies on the $7 \mathrm{H} 10$ agar media, the single cross-over mutants were not viable.

To verify the effective application of the new plasmid in gene regulation, we chose $D d l$ as the reporter gene. The results indicated that the growth of the mutant strains was comparable to wild type strain without anhydrotetracycline. Further, the growth of the mutant strains was abolished when anhydrotetracycline was added as a result of the reduced expression of $D d l$. $D d l$ gene repression was positively correlated with the concentration of anhydrotetracycline. When $2 \mathrm{ng} \mathrm{mL}^{-1}$ anhydrotetracycline was added, the cell wall mass of the mutant decreased by only $14.7 \%$ and there was no difference between the MIC of D-cycloserine to the mutant with or without this effector. However, when the concentration of anhydrotetracycline was increased to $20 \mathrm{ng} \mathrm{mL}^{-1}$, the cell wall mass decreased to $44.9 \%$ and the MIC was 16 times lower. Cell wall mass was only $26.2 \%$ when $200 \mathrm{ng} \mathrm{mL}^{-1}$ anhydrotetracycline was added. After adding $2000 \mathrm{ng} \mathrm{mL}^{-1}$ anhydrotetracycline hardly any colonies were formed and cell wall mass did not changed from $200 \mathrm{ng} \mathrm{mL}^{-1}$. It was speculated that the repression of $D d l$ gene expression through the addition of anhydrotetracycline had reached its limit. Given the low copy number at 200 and $2000 \mathrm{ng} \mathrm{mL} \mathrm{m}^{-1}$ anhydrotetracycline, the MIC of D-cycloserine to the mutant was not determined. The data confirm that we were able to fully repress $\mathrm{D} d l$ gene expression using anhydrotetracycline, and that the amount of Ddl protein correlates to the concentration of anhydrotetracycline added to the culture medium.

This study successfully constructed a new plasmid pMDX for generating stable conditional mutant strains in $M$. tuberculosis using lineated double-stranded DNA electro transformed cells. The application of this plasmid and prospects were demonstrated as we were able to conditionally regulate gene expression of $D d l$. However, this novel approach will need further validation as it is not conform classic molecular manipulation.

\section{Additional file}

Additional file 1. PCR primers used in this study. 


\section{Abbreviations}

ATCC: American type culture collection; Ddl: D-alanine:D-alanine ligase; LB: Luria-Bertani; MIC: minimum inhibitory concentration; Mtb: Mycobacterium tuberculosis; OADC: Oleic Albumin Dextrose Catalase; TB: tuberculosis.

\section{Authors' contributions}

The first author QD wrote this manuscript and participated in all the experiments. YG, YL and CX took part in the design of experiments and experimentation. JM is corresponding author and is responsible for the whole process of experimentation and manuscript subscription. All authors read and approved the final manuscript.

\section{Acknowledgements}

We would like to thank Beijing Chest Hospital, Capital Medical University/Beijing Tuberculosis and Thoracic Tumor Research Institute, Beijing for providing P2 laboratory.

\section{Competing interests}

The authors declare that they have no competing interests.

\section{Availability of data and materials \\ Not applicable.}

\section{Consent for publication}

Not applicable.

\section{Ethics approval and consent to participate}

Not applicable.

\section{Funding}

National Major Scientific and Technological Special Project of "Significant New Drugs Development" (Grant No. 2015ZX09102007-009).

CAMS Initiative for Innovative Medicine (Grant No. 2016-I2M-1-013);

The Fundamental Research Funds for Central Public-interest Scientific Institution (Centre for Tuberculosis) (Grant No. 2017PT31010).

\section{Publisher's Note}

Springer Nature remains neutral with regard to jurisdictional claims in published maps and institutional affiliations.

Received: 8 October 2017 Accepted: 15 January 2018

Published online: 01 February 2018

\section{References}

Allen BW (1998) Mycobacteria. General culture methodology and safety considerations. Methods Mol Biol 101:15-30

Besra GS (1998) Preparation of cell-wall fractions from mycobacteria. Methods Mol Biol 101:91-107

Blair HA, Scott $\sqcup$ (2015) Delamanid: a review of its use in patients with multidrug-resistant tuberculosis. Drugs 75(1):91-100

Blokpoel MC, Murphy HN, O'Toole R, Wiles S, Runn ES, Stewart GR, Young DB, Robertson BD (2005) Tetracycline-inducible gene regulation in mycobacteria. Nucleic Acids Res 33(2):e22-e22

Brown AC, Parish T (2006) Instability of the acetamide-inducible expression vector PJAM2 in Mycobacterium tuberculosis. Plasmid 55(1):81-86

Bruning JB, Murillo AC, Chacon O, Barletta RG, Sacchettini JC (2011) Structure of the Mycobacterium tuberculosis D-alanine:D-alanine ligase, a target of the antituberculosis drug D-cycloserine. Antimicrob Agents Chemother 55(1):291-301

Carroll P, Muttucumaru DG, Parish T (2005) Use of a tetracycline-inducible system for conditional expression in Mycobacterium tuberculosis and Mycobacterium smegmatis. Appl Environ Microbiol 71(6):3077-3084

Cascioferro A, Boldrin F, Serafini A, Provvedi R, Palu G, Manganelli R (2010) Xer site-specific recombination, an efficient tool to introduce unmarked deletions into mycobacteria. Appl Environ Microbiol 76(15):5312-5316
Chalut C, Botella L, de Sousa-D'Auria C, Houssin C, Guilhot C (2006) The nonredundant roles of two $4^{\prime}$-phosphopantetheinyl transferases in vital processes of Mycobacteria. Proc Natl Acad Sci USA 103(22):8511-8516

Chen D, Lin J, Che Y, Liu X, Lin J (2011) Construction of recombinant mercury resistant Acidithiobacillus caldus. Microbiol Res 166(7):515-520

Ehrt S, Guo XV, Hickey CM, Ryou M, Monteleone M, Riley LW, Schnappinger D (2005a) Controlling gene expression in mycobacteria with anhydrotetracycline and Tet repressor. Nucleic Acids Res 33(2):e21

Ehrt S, Guo XV, Hickey CM, Ryou M, Monteleone M, Riley LW, Schnappinger D (2005b) Controlling gene expression in mycobacteria with anhydrotetracycline and Tet repressor. Nucleic Acids Res 33(2):e21-e21

Forti F, Crosta A, Ghisotti D (2009) Pristinamycin-inducible gene regulation in mycobacteria. J Biotechnol 140(3):270-277

Gandotra S, Schnappinger D, Monteleone M, Hillen W, Ehrt S (2007) In vivo gene silencing identifies the Mycobacterium tuberculosis proteasome as essential for the bacteria to persist in mice. Nat Med 13(12):1515-1520

Gomez JE, Bishai WR (2000) whmD is an essential mycobacterial gene required for proper septation and cell division. Proc Natl Acad Sci USA 97(15):8554-8559

Gordhan BG, Parish T (2001) Gene replacement using pretreated DNA. Methods Mol Med 54:77-92

Guo XV, Monteleone M, Klotzsche M, Kamionka A, Hillen W, Braunstein M, Ehrt S, Schnappinger D (2007) Silencing Mycobacterium smegmatis by using tetracycline repressors. J Bacteriol 189(13):4614-4623

Hernandez-Abanto SM, Woolwine SC, Jain SK, Bishai WR (2006) Tetracyclineinducible gene expression in mycobacteria within an animal host using modified Streptomyces tcp830 regulatory elements. Arch Microbiol 186(6):459-464

Hinds J, Mahenthiralingam E, Kempsell KE, Duncan K, Stokes RW, Parish T, Stoker NG (1999) Enhanced gene replacement in mycobacteria. Microbiology 145(Pt 3):519-527

Hodgkinson JW, Ge JQ, Katzenback BA, Havixbeck JJ, Barreda DR, Stafford JL, Belosevic M (2015) Development of an in vitro model system to study the interactions between Mycobacterium marinum and teleost neutrophils. Dev Comp Immunol 53(2):349-357

Kwon YS (2017) Clinical implications of new drugs and regimens for the treatment of drug-resistant tuberculosis. Chonnam Med J 53(2):103-109

Lu P, Villellas C, Koul A, Andries K, Lill H, Bald D (2014) The ATP synthase inhibitor bedaquiline interferes with small-molecule efflux in Mycobacterium smegmatis. J Antibiot (Tokyo) 67(12):835-837

Meng J, Yang Y, Xiao C, Guan Y, Hao X, Deng Q, Lu Z (2015) Identification and validation of aspartic acid semialdehyde dehydrogenase as a new antimycobacterium tuberculosis target. Int J Mol Sci 16(10):23572-23586

Pandey AK, Raman S, Proff R, Joshi S, Kang CM, Rubin EJ, Husson RN, Sassetti CM (2009) Nitrile-inducible gene expression in mycobacteria. Tuberculosis 89(1):12-16

Parish T, Stoker NG (2000) Use of a flexible cassette method to generate a double unmarked Mycobacterium tuberculosis tlyA plcABC mutant by gene replacement. Microbiology 146(Pt 8):1969-1975

Parish T, Mahenthiralingam E, Draper P, Davis EO, Colston MJ (1997) Regulation of the inducible acetamidase gene of Mycobacterium smegmatis. Microbiology 143(Pt 7):2267-2276

Parish T, Gordhan BG, McAdam RA, Duncan K, Mizrahi V, Stoker NG (1999) Production of mutants in amino acid biosynthesis genes of Mycobacterium tuberculosis by homologous recombination. Microbiology 145(Pt 12):3497-3503

Ravishankar S, Ambady A, Ramu H, Mudugal NV, Tunduguru R, Anbarasu A, Sharma UK, Sambandamurthy VK, Ramaiah S (2015) An IPTG inducible conditional expression system for mycobacteria. PLOS ONE 10(8):e0134562

Seeliger JC, Topp S, Sogi KM, Previti ML, Gallivan JP, Bertozzi CR (2012) A Riboswitch-based inducible gene expression system for mycobacteria. PLOS ONE 7(1):e29266

van Helden PD, Victor TC, Warren RM, van Helden EG (2001) Isolation of DNA from Mycobacterium tuberculosis. Methods Mol Med 54:19-30

van Kessel JC, Hatfull GF (2007) Recombineering in Mycobacterium tuberculosis. Nat Methods 4(2):147-152

Zhang G, Gurtu V, Kain SR (1996) An enhanced green fluorescent protein allows sensitive detection of gene transfer in mammalian cells. Biochem Biophys Res Commun 227(3):707-711 